\title{
Desempenho mastigatório em adultos relacionado com a desordem temporomandibular e com a oclusão $* * * * *$
}

\author{
Masticatory performance in adults related to temporomandibular \\ disorder and dental occlusion
}

\author{
Cláudia Maria de Felício* \\ Melissa de Oliveira Melchior** \\ Marco Antônio Moreira Rodrigues da Silva*** \\ Renata Maria dos Santos Celeghini****
}

*Fonoaudióloga. Doutora em Ciências pela Universidade de São Paulo. Professora do Departamento de Oftalmologia, Otorrinolaringologia e Cirurgia de Cabeça e Pescoço - Área Fonoaudiologia da Faculdade de Medicina de Ribeirão Preto da Universidade de São Paulo. Endereço para correspondência: Av. Bandeirantes, 3900 - Ribeirão Preto São Paulo - CEP 14049-900 (efelicio@fmrp.usp.br).

**Fonoaudióloga. Especialista em Motricidade Oral pela Universidade de Ribeirão Preto.

***Cirurgião Dentista. Livre-Docente do Departamento de Odontologia da Faculdade de Odontologia de Ribeirão Preto da Universidade de São Paulo.

****Química. Doutora em Química Analítica pelo Instituto de Química de São Carlos da Universidade de São Paulo.

*****Pesquisa Realizada nas Faculdades de Medicina e de Odontologia de Ribeirão Preto da Universidade de São Paulo.

Artigo de Pesquisa

Artigo Submetido a Avaliação por Pares

Conflito de Interesse: não

Recebido em 18.08.2006.

Revisado em 06.11.2006; 18.04.2007; 29.05.2007.

Aceito para Publicação em 29.05.2007.

\begin{abstract}
Background: temporomandibular disorder and mastication. Aim: to compare subjects who present temporomandibular disorders to a control group considering mastication and to analyze the related variables. Methods: 20 subjects with temporomandibular disorder (TMD group) and 10 controls selection based on clinical examination and anamnesis - responded to a questionnaire on the selfperception of pain severity and presence of noise in the temporomandibular joints, muscle pain, otologic symptoms, headaches, and jaw opening difficulties. The subjects were also submitted to a clinical examination regarding the number of teeth and functional occlusion - measurements of jaw opening and jaw lateral excursions, occlusal interferences, occlusal contacts of the working and nonworking-side, and mastication evaluation. Mastication was evaluated in terms of time needed to eat a stuffed cookie, number of chewing strokes and type (unilateral or bilateral). The maximum force needed at first to break the cookie, verified with a TA-XT2 Texture Analyzer (Stable Micro Systems), was of $4341.8 \mathrm{~g}$. The groups were compared using variance analysis and the correlations between variables were calculated using the Pearson product-moment test. Results: most of the control subjects presented bilateral pattern of mastication, whereas the TMD group tended to present the unilateral pattern. Masticatory type scores and laterality measurements were significantly higher in the control group. The TMD group presented higher means in terms of: age, time of chewing, number of chewing strokes and TMD severity. Chewing time and type were positively correlated with TMD severity and negatively correlated with number of occlusal interferences. Conclusion: in the TMD group, chewing differed from the normal physiological standard. The number of occlusal interferences and the severity of TMD were variables correlated to chewing.
\end{abstract}

Key Words: Temporomandibular Joint; Mastication; Dental Occlusion; Food Analysis.

\section{Resumo}

Tema: desordem temporomandibular e mastigação. Objetivo: comparar sujeitos com desordem temporomandibular a um grupo controle quanto à mastigação e analisar as variáveis relacionadas. Métodos: 20 sujeitos com desordem temporomandibular (grupo com DTM) e 10 do grupo controle, ambos selecionados de acordo com o exame clínico e anamnese, responderam sobre a sua autopercepção de severidade de dor e sons nas articulações temporomandibulares, dor nos músculos, sintomas otológicos, cefaléia e dificuldade para abrir a boca. Foram também submetidos ao exame clínico, considerando o número de elementos dentários presentes e a análise funcional da oclusão - medidas de abertura bucal, excursão lateral da mandíbula, interferências oclusais e contatos oclusais do lado de trabalho e balanceio. A mastigação foi avaliada quanto ao tempo para ingerir, ao número de golpes mastigatórios e ao tipo mastigatório (unilateral ou bilateral), usando um biscoito recheado, cuja força máxima para quebrá-lo no primeiro momento foi de $4341,8 \mathrm{~g}$, como verificado com o auxílio do Texture Analyser TA-XT2 (Stable Micro Systems). Os grupos foram comparados por análise de variância e as correlações entre as variáveis foram calculadas pelo teste produto-momento de Pearson. Resultados: a maioria dos sujeitos do grupo controle apresentou tipo mastigatório bilateral, enquanto que no grupo com DTM houve tendência ao tipo mastigatório unilateral. No grupo controle foram estatisticamente maiores os escores do tipo mastigatório e as medidas de lateralidade. No grupo com DTM foram maiores as médias de idade, o tempo de mastigação, o número de golpes mastigatórios e a severidade da DTM. O tempo e o tipo mastigatório foram correlacionados, respectivamente de modo positivo e negativo, à severidade da DTM e ao número de interferências oclusais. Conclusão: no grupo com DTM a mastigação diferiu do padrão fisiológico normal. O número de interferências oclusais e a severidade da DTM foram as variáveis correlacionadas à mastigação.

Palavras-Chave: Articulação Temporomandibular; Mastigação; Oclusão Dentária; Análise de Alimentos.

Referenciar este material como:

13 FELÍCIO, C. M.; MELCHIOR, M. O.; SILVA M. A. M. R.; CELEGHINI, R. M. S. Desempenho mastigatório em adultos relacionado com a desordem temporomandibular e com a oclusão. Pró-Fono Revista de Atualização Científica, Barueri (SP), v. 19, n. 2, p.151-158, abr.-jun. 2007. 


\section{Introdução}

Estudos têm focalizado a função mastigatória com a finalidade de descrevê-la (Hiiemae et al. 1996) e de propor métodos para avaliá-la (Mioche et al. 2003; Whitaker, 2005), devido à importância no processo digestivo e à relação recíproca com os componentes do sistema estomatognático.

A força, os movimentos, o tempo e o tipo de mastigação são aspectos influenciáveis por variáveis como: a morfologia, o estado de saúde das estruturas orofaciais, a capacidade funcional muscular e a articular, além das características dos alimentos (Mazzetto et al. 2002; Mioche et al. 2003; Engelen et al. 2005; Cattoni, 2005).

Pelo fato de indivíduos portadores de desordem temporomandibular (DTM) freqüentemente apresentarem limitações na função mastigatória (Felício et al. 2002; Sato et al. 2002), eles têm sido comparados à grupos controle. Embora a maioria dos estudos aponte uma prevalência de mastigação unilateral entre os sujeitos com DTM (Miyawaki et al. 2004; Felício et al. 2005), nem sempre foi constatado diferenças significantes em relação ao grupo controle (Souza et al. 2005), principalmente quando foram selecionados indivíduos que também apresentavam alterações oclusais, mas não DTM (Felício et al. 2002).

Segundo estudos prévios, em sujeitos com oclusão adequada e sem disfunção, o processo ocorre com atividade simétrica entre os músculos masseter e temporal anterior esquerdo e direito (Ferrario et al. 2000). Porém, em sujeitos com interferências oclusais do lado de balanceio (Nishigawa et al. 1997) e com mordida cruzada unilateral (Pizzol, 2004) o padrão de contração muscular alterado e assimétrico tem sido observado por meio de eletromiografia e palpação, durante a mastigação. Também, durante o apertamento em máxima intercuspidação habitual, sujeitos com diagnóstico de DTM apresentaram assimetria maior que sujeitos saudáveis (Alajbeg et al. 2003).

Os problemas oclusais são relacionados aos sinais e sintomas da desordem temporomandibular (DTM), podendo afetar a função mastigatória e propiciar assimetria funcional do sistema estomatognático (Hatch et al. 2001; Miyawaki et al. 2004). Contudo, a grande variabilidade de movimentos mastigatórios entre os sujeitos sugere que, os efeitos dos impulsos periféricos sobre os movimentos mastigatórios diferem de indivíduo para indivíduo e variáveis oclusais podem ou não influenciar as respostas motoras dos movimentos mandibulares durante a mastigação, dependendo do padrão de movimento mastigatório individual desenvolvido (Ogawa et al. 2001).

Uma questão importante é que a função mastigatória pode ser afetada por uma complexa interação de efeitos diretos e indiretos e em muitos estudos eles são considerados de modo isolado (Hatch et al. 2001; Salgado, 2002).

O objetivo geral da presente pesquisa foi comparar um grupo de pacientes com desordem temporomandibular a um grupo controle quanto à função mastigatória e analisar as possíveis variáveis relacionadas. Os objetivos específicos foram:

. comparar os grupos quanto às seguintes variáveis: idade, tempo de mastigação, número de golpes e tipo mastigatório, severidade da DTM, medidas dos movimentos excursivos da mandíbula, número de interferências oclusais dos lados de balanceio e de trabalho e número de dentes;

. analisar a correlação entre as variáveis descritas.

\section{Método}

Todos os participantes foram informados dos objetivos do estudo e eles assinaram o termo de consentimento para participar. O projeto foi aprovado pelo Comitê de Ética em Pesquisa da FORP-USP - processo n ${ }^{\circ}$. 99.1.225.58.4.

Participaram da pesquisa 20 sujeitos com Desordem Temporomandibular (DTM) que procuraram por tratamento para o problema, e 10 sujeitos sem queixas de DTM os quais compuseram o Grupo controle (GC).

\section{Critérios de inclusão}

Grupo com DTM: os sujeitos deveriam apresentar no exame clínico odontológico sinais e sintomas de DTM como: presença de dor nos músculos mastigatórios e/ou na articulação temporomandibular (ATM) durante a função mandibular e à palpação, limitação ou desvios nos movimentos mandibulares, ruídos na ATM, e oclusão estática ou dinâmica anormal.

Grupo sem DTM: os sujeitos deveriam ser livres de sinais e sintomas de DTM, com base no mesmo modelo de diagnóstico, independente da condição oclusal.

Critérios de Exclusão: foram excluídos dos grupos sujeitos usuários de prótese total superior, inferior ou ambas, bem como portadores de distúrbios neurológicos, ou que tivessem sofrido tumores ou traumas na região de cabeça e pescoço. 
Dos grupos com DTM também foram excluídos sujeitos que estivessem em quadro agudo de dor que os impedisse de mastigar.

Procedimentos

Foram usados para obter dados sobre a mastigação: câmara de vídeo JVC-GR-AX-720, fitas de vídeo cassete $8 \mathrm{~mm}$, cronômetro digital (Technos), protocolos impressos e biscoitos recheados.

Com a finalidade de determinar precisamente as características do alimento empregado, a medida de textura dos biscoitos foi realizada pela avaliação do parâmetro de dureza. As análises foram realizadas no equipamento Texture Analyser TAXT2 (Stable Micro Systems) com "probe" em forma de lâmina (HDP/BS Knife Edge). Foram analisadas 10 amostras de biscoito de chocolate marca Bonnoâ; cada amostra foi removida da embalagem no momento do teste. As condições de análise foram: modo de análise força em compressão; velocidade pré-teste $2,0 \mathrm{~mm} / \mathrm{s}$, velocidade teste $2,0 \mathrm{~mm} / \mathrm{s}$ e velocidade pós-teste $10,0 \mathrm{~mm} / \mathrm{s}$; força $20 \mathrm{~g}$ e distância $20 \mathrm{~mm}$. A medida de textura dos biscoitos foi realizada pela avaliação da força máxima no primeiro momento de quebra do biscoito. Neste ponto, o biscoito é fraturado em dois pedaços, a força seguinte é substancialmente reduzida e a penetração continua da lâmina quebra o biscoito em pequenos pedaços. $\mathrm{O}$ valor da força máxima detectado foi de $4341,8 \mathrm{~g}$ com coeficiente de variação de $11 \%$.

Os sujeitos responderam a um questionário a respeito dos sinais e sintomas relacionados a DTM (dor na ATM, dor na face, ruído na ATM, cefaléia, dor cervical, desconforto na mordida, sensibilidade nos dentes, sintomas auditivos, dificuldade na abertura bucal, para deglutir e para falar), que admitia apenas respostas afirmativas ou negativas. Em seguida atribuíram escores de zero (ausência de dor) a sete (dor muito intensa) à intensidade da dor na região crânio-mandibular. O grau de severidade da DTM de cada sujeito foi estabelecido pela somatória do número de sintomas, multiplicado pelo escore de dor por ele atribuído. O questionário pode ser aplicado a sujeitos assintomáticos, pois comporta o grau zero de DTM.

Na avaliação da oclusão foram registrados: o número de elementos dentários presentes; de interferências oclusais do lado de balanceio e do lado de trabalho, isto é contatos dos dentes posteriores na excursão lateral da mandíbula; e as medidas excursivas da mandíbula - máxima abertura bucal, lateralidade direita e esquerda, partindo da máxima intercuspidação habitual (MIH).

Para avaliação da mastigação, os sujeitos se sentaram em uma cadeira comum, com os pés apoiados no chão. Um biscoito recheado foi oferecido e os sujeitos foram instruídos a mastigar de maneira habitual, enquanto as imagens eram registradas em vídeo-cassete. $\mathrm{O}$ teste mastigatório foi realizado individualmente e a análise dos vídeos foi realizada posteriormente por dois examinadores independentes. Para analisar o tempo para consumir o biscoito, o cronômetro foi acionado sempre após a colocação do alimento na cavidade oral e a oclusão labial, sendo paralisado quando ocorria a deglutição final de cada porção. Para analisar o tipo mastigatório foi considerado se a trituração era bilateral alternada, bilateral simultânea, unilateral crônica (95\% do tempo de um mesmo lado da cavidade oral) ou preferência mastigatória unilateral ( $66 \%$ de um mesmo lado) (Felício, 2002), ou ainda se a mastigação realizada com os dentes anteriores (anterior). Posteriormente, de acordo com as porcentagens obtidas, foram atribuídos escores da seguinte maneira: trituração bilateral alternada (3), bilateral simultânea (2), preferência mastigatória unilateral (1), unilateral crônica ou anterior (0).

O número de golpes mastigatórios foi contado a cada elevação da mandíbula com intercuspidação, isto é, ao ocorrer o contato dente-alimento-dente.

\section{Análise dos dados}

Para a comparação entre os grupos foi realizada a análise de variância, considerando os grupos como variáveis dependente e os outros fatores como variáveis independentes. O teste de correlação produto-momento de Pearson foi aplicado para investigar as possíveis correlações entre as variáveis.

\section{Resultados}

Os grupos analisados de acordo com o gênero foram compostos da seguinte maneira, Grupo com DTM (GDTM), três homens e 17 mulheres e no GC, quatro homens e seis mulheres.

A análise de variância revelou diferenças significantes entre os grupos GDTM e GC quanto à idade, o tempo de mastigação, o tipo mastigatório, a severidade da desordem temporomandibular e as medidas dos movimentos de lateralidade direita e esquerda da mandíbula. No GC foram estatisticamente maiores as médias dos escores 
do tipo mastigatório e das medidas de lateralidade direita e esquerda, em comparação ao GDTM. No GDTM foram maiores as médias de idade, de tempo de mastigação, de número de golpes mastigatórios e de severidade da DTM. Na Tabela 1 constam as médias e os resultados da análise de variância.

TABELA 1. Comparação dos grupos GDTM e GC: médias e resultados da análise de variância.

\begin{tabular}{|c|c|c|c|c|}
\hline Variáveis & GDTM & GC & Valor de F & Valor de $\mathrm{P}$ \\
\hline idade (em anos) & 37,15 & 24,1 & $7,909 *$ & 0,009 \\
\hline tempo de mastigação (em segundos) & 116,511 & 78,964 & $11,40 *$ & 0,002 \\
\hline tipo mastigatório & 1,55 & 2,6 & $5,822 *$ & 0,022 \\
\hline número de golpes & 66,25 & 54,3 & 3,037 & 0,092 \\
\hline severidade da DTM & 48,8 & 1,3 & $48,236^{*}$ & 0,000 \\
\hline abertura bucal (em milímetros) & 48,15 & 50,2 & 0,522 & 0,476 \\
\hline lateralidade direita (em milímetros) & 4,5 & 7,4 & $11,102 *$ & 0,002 \\
\hline lateralidade esquerda (em milímetros) & 4,5 & 7,9 & $17,139 *$ & 0,000 \\
\hline interferência balanceio & 1,3 & 0,6 & 1,675 & 0,206 \\
\hline interferência trabalho & 1,9 & 1,1 & 1,905 & 0,178 \\
\hline Número de dentes & 30,55 & 31,6 & 3,718 & 0,064 \\
\hline
\end{tabular}

Legenda: * diferenças significantes entre os grupos; $\mathrm{p}=$ probabilidade de igualdade entre as amostras.

Correlação entre as variáveis

O tipo mastigatório foi negativamente correlacionado ao número de golpes mastigatórios, à severidade da DTM e ao número de interferências oclusais do lado de balanceio. O tempo de mastigação foi positivamente correlacionado às mesmas variáveis e ao número de interferências oclusais do lado de trabalho.

A idade dos sujeitos foi correlacionada positivamente à severidade da DTM e negativamente à medida de lateralidade direita. A severidade da DTM também foi correlacionada negativamente com a medida de lateralidade esquerda.

A medida de lateralidade direita foi positivamente correlacionada com a medida de lateralidade esquerda e negativamente com o número de interferências oclusais do lado de balanceio. $\mathrm{O}$ número de dentes foi positivamente correlacionado à medida de lateralidade esquerda. Os índices de correlação (r de Pearson) e os níveis de significância estão na Tabela 2 , bem como as correlações entre outras variáveis.

TABELA 2. Valores de $\mathrm{r}$ da correlação produto-momento de Pearson e níveis de significância $(\mathrm{N}=30)$.

\begin{tabular}{|c|c|c|c|c|c|c|c|c|c|c|}
\hline & \multirow[b]{2}{*}{ Idade } & \multicolumn{3}{|c|}{ Mastigação } & \multirow{2}{*}{$\begin{array}{c}\text { DTM } \\
\text { Severidade }\end{array}$} & \multirow[b]{2}{*}{ Abertura } & \multicolumn{2}{|c|}{ Lateralidade } & \multicolumn{2}{|c|}{ Interferência } \\
\hline & & Tempo & Tipo & $\mathrm{N}^{\circ}$ Golpes & & & Direita & Esquerda & Balanceio & Trabalho \\
\hline tempo de mastigação & 0,30 & & & & & & & & & \\
\hline tipo mastigatório & $-0,17$ & $-0,35$ & & & & & & & & \\
\hline número de golpes & 0,25 & $0,68 * * *$ & $-0,36^{*}$ & & & & & & & \\
\hline severidade da DTM & $0,41^{*}$ & $0,46 * *$ & $-0,38 *$ & 0,17 & & & & & & \\
\hline abertura & $-0,29$ & $-0,15$ & 0,26 & $-0,15$ & $-0,25$ & & & & & \\
\hline lateralidade direita & $-0,42 *$ & $-0,28$ & 0,33 & $-0,21$ & $-0,33$ & 0,34 & & & & \\
\hline lateralidade esquerda & $-0,22$ & $-0,31$ & 0,26 & $-0,07$ & $-0,59 * * *$ & $-0,00$ & $0,53 * *$ & & & \\
\hline interferência balanceio & 0,11 & $0,45^{*}$ & $-0,38^{*}$ & 0,23 & 0,27 & $-0,02$ & $-0,45^{*}$ & $-0,40$ & & \\
\hline interferência trabalho & 0,32 & $0,47 * *$ & $-0,13$ & 0,26 & 0,23 & 0,18 & $-0,29$ & $-0,25$ & $0,54 * *$ & \\
\hline número de dentes & $-0,26$ & $-0,13$ & 0,13 & 0,04 & $-0,31$ & $-0,01$ & 0,26 & $0,49 * *$ & $-0,01$ & 0,01 \\
\hline
\end{tabular}

Legenda: Significante ao nível de $5 \%=* ; 1 \%=* * ; 0,01 \%=* * *$ 


\section{Discussão}

Quando um alimento é levado à boca, este passa uma série de eventos até ser totalmente consumido, como transporte para a área pós-canina, trituração, transporte para a região dos pilares das fauces. A mastigação só é concluída após a fase de liberação, em que ocorre um período de coleta dos pedaços de alimento que restaram no vestíbulo bucal e a deglutição final (Hiiemae et al. 1996). Por esta razão, neste estudo, ao analisar o tempo de mastigação foi considerada também a deglutição do alimento.

Também, foi determinada a textura do alimento, considerando que muitas divergências entre estudos que investigaram a mastigação de indivíduos com e sem DTM podem ser decorrentes do tipo de alimento utilizado, pois o processo mastigatório sofre influência das características do alimento (Douglas, 2002; van der Bilt, 2002; Mioche et al. 2003; Engelen et al. 2005; Berretin-Felix et al. 2005).

No presente estudo, a maioria dos sujeitos do GC apresentou tipo mastigatório bilateral, enquanto que no GDTM houve tendência ao tipo mastigatório unilateral. Os resultados são condizentes com a literatura (Miyawaki et al. 2004; Felício et al. 2005). Estudos anteriores demonstraram a concordância entre o método clínico e o eletromiográfico para a determinação do lado da mastigação (Christensen, Raude, 1985; Pignataro Neto et al. 2004), bem como entre a utilização de cronômetro e a eletromiografia para o registro da duração do ato mastigatório (Arrais et al. 2004).

A média de idade do GC foi significantemente menor que do GDTM, mas esta variável não foi correlacionada ao tempo e ao tipo mastigatório. Segundo a literatura, sujeitos com DTM com idade acima de 50 anos apresentam escores de habilidade mastigatória significantemente mais baixos do que sujeitos com idade abaixo de 40 anos (Kurita et al. 2001). Contudo, a idade em si pode não ser um fator determinante para ocorrência de alterações na função mastigatória, desde que haja manutenção da saúde dentária e muscular. Alterações no padrão mastigatório foram observadas, quando os efeitos da idade foram associados às condições dentárias em termos de número de unidades funcionais (Kohyama et al. 2003), que apresenta relação com o nível de atividade muscular mastigatória e é considerado o melhor preditivo na determinação da performance mastigatória (Hatch et al. 2001; Ferrario et al. 2002).

Assim, com o passar dos anos, a necessidade de diferenciação entre o que é doença e o processo de envelhecimento é necessária à tomada de conduta (Nisa e Castro et al. 2004), pois os fatores negativos que isoladamente não afetariam de forma significante o funcionamento do sistema podem passar a afetá-lo, devido a um efeito cumulativo dos mesmos (Hatch et al. 2001; Salgado, 2002). Isso talvez ofereça suporte à correlação positiva entre a idade e a severidade da DTM verificada.

Falhas dentárias e o uso de próteses foram anteriormente associados à assimetria dos músculos masseteres e temporais em contração, observados à palpação, que por sua vez poderia ser justificado pela predominância do tipo mastigatório unilateral (Monteiro et al. 2005). No presente estudo o número de dentes não foi correlacionado de modo significante com o tempo de ingestão, o tipo mastigatório, a severidade da DTM e a idade dos sujeitos. Provavelmente, porque o número de dentes ausentes era pequeno e foram excluídos dos grupos sujeitos usuários de prótese total superior, inferior ou ambas.

A duração do ato mastigatório nem sempre apresenta diferença significante entre indivíduos com e sem DTM (Berretin-Felix et al. 2005), mas de acordo com os resultados do presente estudo, o tempo de mastigação foi maior para os sujeitos do GDTM, concordando com estudo anterior (Felício et al. 2002), enquanto os escores quanto ao tipo mastigatório foram menores. Os resultados de ambas as variáveis - tempo e tipo - podem ser interpretados como refletindo o desempenho funcional do sistema estomatognático.

Houve correlação negativa entre o tipo mastigatório e o número de interferências oclusais, e correlação positiva entre o tempo de mastigação e a severidade da DTM. Contatos dos dentes posteriores na excursão lateral da mandíbula, isto é, interferências oclusais, podem aumentar a carga funcional sobre as articulações temporomandibulares (ATMs), por alterar a coordenação muscular entre os lados direito e esquerdo (Nishigawa et al. 1997; Ferrario et al. 2000; Ferrario et al. 2003), e a estabilidade da movimentação mandibular durante a mastigação apresenta íntima relação com a eficiência mastigatória (Unno, et al. 2005).

Assim o maior número de interferências oclusais e a severidade da DTM parecem prejudicar o desempenho funcional mastigatório, levando a um tipo diferente da fisiologia normal (bilateral alternado) e aumentando o tempo necessário para o processamento e ingestão do alimento. Isto pode ser interpretado como um esforço para prevenir 
um desconforto e uma hesitação para escolher uma área onde o biscoito poderia ser mais bem triturado, isto é, a área de maior eficiência mastigatória (Felício et al. 2002).

A correlação positiva do número de golpes mastigatórios com o tempo e negativa com o tipo mastigatório são condizentes com as explicações anteriores. Todavia, provavelmente, a evitação de estímulos nociceptivos e as modificações no esquema proprioceptivo afetem a adaptabilidade e a coordenação dos movimentos mandibulares.

Sendo assim, é interessante considerar que as interferências oclusais também podem ser conseqüências de uma modificação da posição oclusal, secundária à presença de dor articular e/ ou muscular (Fujii, 2002).

Diferenças significantes na amplitude de movimentos mandibulares foram observadas entre o GC e GDTM, em acordo com estudos anteriores (Celic et al. 2004; Hansdottir e Bakke, 2004). Medidas de lateralidade foram negativamente correlacionadas com o número de interferências oclusais e com a severidade da DTM. Não houve correlações significantes destes movimentos com resultados quanto ao tipo mastigatório (Felício et al. 2005) e ao tempo de mastigação.

Foi demonstrado previamente, que os movimentos mastigatórios humanos, relacionados à guia oclusal, diferem de acordo com os padrões de movimentos adquiridos individualmente (mais horizontais ou mais verticais), o que implica em respostas motoras variadas, de acordo com o potencial funcional de cada indivíduo (Ogawa et al. 2001).

Injúrias do sistema estomatognático podem influenciar a capacidade adaptativa e determinar um prejuízo no padrão de trituração (tipo), afetando o desempenho mastigatório (Hatch et al. 2001; Kurita, et al. 2001; Mazzetto et al. 2002; Sato et al. 2003; Pizzol, 2004; Cattoni, 2005). Uma marcante deficiência funcional foi previamente associada às diferenças encontradas em sujeitos com dor articular crônica, como menor limiar de dor à pressão, menor abertura bucal, maior duração de ciclos mastigatórios, menor velocidade de fechamento e menor força de mordida, quando comparados a sujeitos saudáveis. Tal deficiência foi atribuída a um possível reflexo adaptativo e à hipoatividade prolongada dos músculos mastigatórios (Hansdottir e Bakke, 2004).

As compensações realizadas para executar a função mastigatória em condições adversas, mesmo que de modo reflexo, nem sempre são eficazes e podem contribuir com a progressão da DTM a longo prazo.

\section{Conclusão}

O presente estudo permitiu concluir que houve diferenças entre os grupos comparados. As médias dos escores do tipo mastigatório e das medidas de lateralidade direita e esquerda foram maiores no GC, enquanto no GDTM foram maiores as médias de idade, do tempo de mastigação, do número de golpes mastigatórios e da severidade da DTM.

O número de interferências oclusais e a severidade da DTM foram as variáveis correlacionadas à mastigação. A tendência foi a seguinte: quanto maior o número de interferências oclusais e a severidade da DTM, maior o tempo de mastigação e menor o escore quanto ao tipo mastigatório, ou seja, mais distante do padrão fisiológico normal. 


\section{Referências Bibliográficas}

ALAJBEG, I. Z.; VALENTIC-PERUZOVIC, M.; ALAJBEG, I.; ILLES, D. Influence of occlusal stabilization splint on the asymmetric activity of masticatory muscles in patients with temporomandibular dysfunction. Coll. Antropol., Zagreb, v. 27, n. 1, p. 361-371, jun. 2003.

ARRAIS, R. D.; GENARO, K. F.; SAMPAIO, A. C. M. Função mastigatória em indivíduos normais: duração do ato e do ciclo mastigatório. In: SIMPÓSIO INTERNACIONAL DE INICIAÇÃO CIENTÍFICA, 12., 2004, Ribeirão Preto. Anais... São Paulo: 2004. p. 22-23.

BERRETIN-FELIX, G.; GENARO, K. F.; TRINDADE, I. E. K.; TRINDADE JÚNIOR, A. S. Masticatory function in temporomandibular dysfunction patients: eletromiographic evaluation. J. Appl. Oral. Sci., Bauru, v. 13, n. 4, p. 360-365, out.-dez. 2005.

CATTONI, D. M. Alterações da mastigação e deglutição. In: FERREIRA, L. P.; BEFI-LOPES, D. M.; LIMONGI, S. C. O.; PUPO, A. C.; FURKIM, A. M.; CHIARI, B. M.; BIANCHINI, E. M. G.; RAMOS, S. M. Tratado de fonoaudiologia. 1. ed. reimpressão. São Paulo: Roca, 2005. cap. 24, p. 277-291.

CELIC, R.; JEROLIMOV, V.; KNEZOVIC ZLATARIC, D. Relationship of slightly limited mandibular movements to temporomandibular disorders. Braz. Dent. J., Ribeirão Preto, v. 15, n. 2, p. 151-154, 2004.

CHRISTENSEN, L. V.; RADUE, J. T. Lateral preference in mastication: an electromyographic study. J. Oral. Rehabil., Oxford, v. 12, n. 5, p. 429-434, sep. 1985.

DOUGLAS, C. R. Funções gerais desenvolvidas pela boca. In: DOUGLAS, C. R. Tratado de fisiologia aplicada à fonoaudiologia. São Paulo: Robe, 2002. p. 285-288.

ENGELEN, L.; FONTIJIN-TEKAMP, A.; VAN DER BILT, A. The influence of product and oral characteristics on swallowing. Arch. Oral. Biol., Oxford, v. 50, n. 8, p. 739-746, aug. 2005

FELÍCIO, C. M.; FERNANDES, R. S. M.; RODRIGUES DA SILVA, M. A. M. Investigação da relação entre o tipo mastigatório e o limite de movimento lateral da mandíbula. R. Soc. Bras. Fonoaudiol., São Paulo, v. 10, n. 2, p. 7176, maio-jun. 2005

FELÍCIO, C. M.; MAZZETTO, M. O.; DOS SANTOS, C. P. A. Masticatory behavior in individuals with temporomandibular disorders. Minerva Stomatol., Torino, v. 51, n. 4, p. 111-120, apr. 2002.

FERRARIO, V. F.; SERRAO, G.; DELLAVIA, C.; CARUSO, E.; SFORZA, C. Relationship between the number of occlusal contacts and masticatory muscle activity in healthy young adults. Cranio, Chattanooga, v. 20, n. 2, p. 91-98, sept. 2002.

FERRARIO, V. F.; SFORZA, C.; COLOMBO, A.; CIUSA, $\mathrm{V}$. A electromiographic investigation of masticatory muscles symmetry in normo-occlusion subjects. J Oral Rehabil., Oxford, v. 27, n. 1, p. 33-40, jan. 2000.

FERRARIO, V. F.; SFORZA, C.; DELlAVIA, C.; TARTAGLIA, G. M. Evidence of an influence of asymmetrical occlusal interferences on the activity of the sternocleidomastoid muscle. J. Oral. Rehabil., Oxford, v. 30, n. 1, p. 33-40, jan. 2003.
FUJII, T. Occlusal conditions just after the relief of temporomandibular joint and masticatory muscle pain. $J$. Oral. Rehabil., Oxford, v. 29, n. 4, p. 323-329, apr. 2002.

HANSDOTTIR, R.; BAKKE, M. Joint tenderness, jaw opening, chewing velocity, and bite force in patients with temporomandibular joint pain and matched healthy control subjects. J. Orofac. Pain., Carol Stream, v. 18, n. 2, p. 108-113, spring. 2004.

HATCH, J. P.; SHINKAI, R. S. A.; SAKAI, S.; RUGH, J. D.; PAUNOVICH, E. D. Determinants of masticatory performance in dentate adults. Arch. Oral. Biol., Oxford, v. 46, n. 7, p. 641-648, jul. 2001.

HIIEMAE, K. M.; HEAT, M. R.; HEAT, G.; KAZAZOGLU, E.; MURRAY, J.; SAPPER, D.; HAMBLET, K. Natural bites, food consistency and feeding behavior in man. Arch. Oral. Biol., Oxford, v. 41, n. 2, p. 175-189, feb. 1996.

KOHYAMA, K.; MIOCHE, L.; BOURDIOL, P. Influence o age and dental status on chewing behavior studied by EMG recordings during consumption of various food samples, Gerodontol., Oxford, v. 20, n. 1, p. 15-23, jul. 2003.

KURITA, H.; OHTSUKA, A.; KURASHINA, K.; KOPP, S. Chewing ability as a parameter for evaluating the disability of patientes with temporomandibular disorders. J. Oral. Rehabil., Oxford, v. 28, n. 5, p. 463-465, may 2001.

MAZZETTO, M. O.; NASCIMENTO, G.; GOMES, N. M. S. Estudo da prevalência das alterações das funções estomatognáticas em pacientes com disfunção temporomandibular. J. Bras. Fonoaudiol., Curitiba, v. 3, n. 11 , p. 140-147, abr.-jun. 2002.

MIOCHE, L.; BOURDIOL, P.; MONIER, S. Chewing behavior and bolus formation during mastication of meat with different textures. Arch. Oral. Biol., Oxford, v. 48, n. 3, p. 193-200, mar. 2003.

MIYAWAKI, S.; TANIMOTO, Y.; ARAKI, Y.; KATAYAMA, A.; KUBOKI, T.; TAKANOYAMAMOTO, T. Movement of the lateral and medial poles of the working condyle during mastication in patients with unilateral posterior crossbite. Am. J. Orthod. Dentofac. Orthop., St. Louis, v. 126, n. 5, p. 549-554, nov. 2004.

MONTEIRO, M. P.; CARNEIRO, F. P; FELIPE, N. A. P.; MOTTA, A. R. Mastigação e dispepsia funcional: um novo campo de atuação. R. Cefac, São Paulo, v. 7, n. 3, p. 340347, jul.-set. 2005

NISA E CASTRO, S. A. F.; HARTMANN, A. C. V. C.; SANTOS, A. C. Medicamentos associados a xerostomia e a distúrbios das funções orofaciais em idosos. $R$. Fonoaudiol. Bras., Brasília, v. 5, n. 1, p. 1-3, dez. 2004. NISHIGAWA, K.; NAKANO, M.; BANDO, E. Study of jaw movement and masticatory muscle activity during unilateral chewing with and without balancing side molar contacts. J. Oral. Rehabil., Oxford, v. 24, n. 9, p. 691696, sept. 1997. 
OGAWA, T.; OGAWA, M.; KOYANO, K. Different responses of masticatory movements after alteration of occlusal guidance related to individual movement pattern. J. Oral. Rehabil., Oxford, v. 28, n. 9, p.830-841, sept. 2001.

PIGNATARO NETO, G.; BERZIN, F.; RONTANI, R. M. P. Identificação do lado de preferência mastigatória através do exame eletromiográfico comparado ao visual. $R$. Dent. Press. Ortodon. Ortopedi. Fac., Maringá, v. 9, n. 4, p. 7785, jul.-ago. 2004.

PIZZOL, K. E. D. C. Influência da mastigação unilateral no desenvolvimento da assimetria facial. R. Uniara, Araraquara, n. 15, p. 215-222, sem 1. 2004.

SALGADO, J. M. Nutrição na $3^{a}$ idade. In: BRUNETTI R. F.; MONTENEGRO, F. L. B. Odontogeriatria: noções de interesse clínico. São Paulo: Artes Médicas, 2002. p. $61-70$.

SATO, S.; FUEKI, H.; SATO, H.; SUEDA, S.; SHIOZAKI, T.; KATO, M.; OHYAMA, T. Validity and reliability of a newly developed method for evaluating masticatory function using discriminant analysis. J. Oral. Rehabil., Oxford, v. 30, n. 2, p. 146-151, feb. 2003.
SATO, S.; FUMIKO, N., KATSUTOSHI, M. Natural course of nonreducing disc displacement of the temporomandibular joint: changes in chewing movement and masticatory efficiency. J. Oral. Maxillofac. Surg., v. 60 , n. 8, p. 867-872, aug. 2002.

SOUZA, D. R.; SALVAT, R. P.; BIANCHINI, E. M. G.; ASSÊNCIO-FERREIRA, V. J. Características mastigatórias em portadores de disfunção temporomandibular-estudo comparativo. R. Soc. Bras. Fonoaudiol., v. 10, n. 3, p. 155-160, jul.-set. 2005.

UNNO, M.; SHIGA, H.; KOBAYASHI, Y. The relationship between masticatory path pattern and masticatory efficiency in gumi-jelly chewing. Nippon Hotetsu Shika Gakkai Zasshi., Tokyo, v. 49, n. 1, p. 65-73, feb. 2005.

VAN DER BILT A. Human oral function: a review. Braz. J. Oral. Sci., Piracicaba, v. 1, n. 1, p.7-18, abr.-jun. 2002.

WHITAKER, M. E. Função mastigatória: proposta de protocolo de avaliação clínica. 2005. 97 f. Dissertação (Mestrado em Distúrbios da Comunicação) - Hospital de Reabilitação de Anomalias Craniofaciais, Universidade de São Paulo, Bauru. 